\title{
Invención dentro del curriculum: sugerencias de un innovador
}

\author{
Invention within the curriculum: suggestions of an innovator
}

\begin{abstract}
Erich Kunhardt ${ }^{\mathrm{a}}$
Cómo citar: Kunhardt, E. (2020). Invención dentro del curriculum: sugerencias de un innovador. Ciencia y Educación, 3(3), 43-52. Doi: https://doi.org/10.22206/cyed.2019.v3i3.pp43-52
\end{abstract}

Esta conferencia trata sobre la invención en el currículo, un tema de gran controversia. Mi abordaje se organizará en torno a tres ámbitos; primero, un re-examen de los propósitos de la educación, preguntándonos ¿cuál es el objetivo?; en segundo lugar, y en función de tales propósitos, me referiré a la cuestión de proveer los medios para el desarrollo intelectual de los educandos y, como parte de esto, lo que denomino el currículo funcional; por último, abordaré el rol del profesor en el proceso educativo, comentando características específicas necesarias en los docentes para, finalmente, ofrecer algunos ejemplos de actividades.

Al reflexionar sobre los propósitos de la educación, parto de constatar que vivimos en contextos en los que existen fuertes presiones, no necesariamente visibles o explícitas, que promueven la homogeneidad entre nosotros e impulsan a que seamos como todo el mundo. Cuando actuamos sometidos a esas presiones es como si quisiéramos que todos probaran solo helados de vainilla; como si preparáramos para todo el mundo un jugo de concentrado de limón en polvo, y todos, entonces, debiéramos beber de ese mismo jugo. Mi invitación es a que no bebamos de ese jugo, a que no nos conformemos con probar exclusivamente helado de vainilla, que abramos nuestras mentes, pensemos en la diversidad y en alternativas, y nos dispongamos a explorar.

\section{¿Cuál es el objetivo de la educación?}

¿Y qué vamos a explorar? Propongo que examinemos el propósito de la educación, que nos preguntemos: ¡cuál es su objetivo? Por lo general nosotros como educadores lo que hacemos es enfocarnos en los contenidos que los estudiantes deben manejar, en cuáles son las "materias" que deben tomar y empezamos a hacer una lista de cotejo de esas "materias". De esa manera, nos ocupamos de habilidades que los estudiantes deben adquirir (multiplicar, despejar ecuaciones, aplicar fórmulas), de los niveles en los que tales habilidades deben demostrarse (las ecuaciones

\footnotetext{
a El Dr. Erich Kunhardt, fallecido en New York en 2014, nació en Montecristi, República Dominicana en 1949. Físico, profesor universitario, investigador, inventor y gestor universitario de gran reconocimiento. Murió siendo profesor de Física Aplicada del Polytechic Institute of New York, hoy New York University Tandon School of Engineering, institución en que había sido Provost. En el año 2007 el Instituto Tecnológico de Santo Domingo (INTEC), le reconoció sus méritos académicos otorgándole un Doctorado Honoris Causa en Ciencias. La conferencia que publicamos con autorización de sus familiares, es una publicación póstuma necesaria. Fue dictada originalmente a profesores y funcionarios de INTEC en el ańo 2010. En ella se refleja su propuesta educativa, la cual refleja la preocupación curricular de un científico, que todavía nos hace pensar.

Correo-e: cienciayeducacion@intec.edu.do
} 
que deben manejar) y también de las informaciones e incluso los libros que deben conocer y leer. En otras palabras, nos remitimos a los contenidos.

Siguiendo ese patrón de pensamiento concebimos el tránsito de los estudiantes por la universidad como uno en el que llegan con sus intelectos poco desarrollados, empiezan a subir los escalones y a hacer su esfuerzo, y cuando salen de la universidad, su intelecto se ha desarrollado y tienen en su mente unos contenidos que han aprendido. Aquí la pregunta clave es, ¿qué desarrolló y con qué hemos provisto su intelecto, su mente? Decimos que el objetivo de ir a la universidad es el cultivo de la mente, pero los invito a explorar y a cuestionar qué es lo que estamos cultivando en esas mentes. Porque, por lo general, damos por supuestos los propósitos y no nos preguntamos ¡cuál es el objetivo de todo esto? Y es de esto último que quisiera que reflexionáramos un poco al iniciar esta plática.

¿Cuál es el objetivo? Para ayudarme en mi propuesta de respuesta a esta pregunta, y para ser sugerente respecto de la exploración y los cuestionamientos a los que les invito, quiero apoyarme en Jacob Bronouski, un matemático de Cambridge, quien en The Origins of Knowledge and Imagination, un libro en el que recopiló cuatro conferencias que impartió sobre ese tema, podemos leerle diciendo que:

Vamos haciendo ciencia exactamente de la misma manera mecanicista porque esa es la única estrategia por medio de la cual progresamos (...). No, no conocemos otra manera de hacer ciencia. Pero está totalmente claro que ahora tenemos una concepción sobre qué es el universo diferente de la de, digamos, la que Newton compartió con Leibniz. Debemos darnos cuenta que la conectividad total de la naturaleza es algo que no puede ser imaginado en los sistemas axiomáticos.

Quiero resaltar la paradoja que se expresa en esta cita de Bronowsky: la ciencia avanza como un mecanismo y "no conocemos otra manera de hacer ciencia", pero, al mismo tiempo, queda totalmente claro que hoy tenemos una concepción diferente sobre qué es el universo de la que tenían Newton y Leibniz siglos atrás. Y aquí subrayo una afirmación muy importante en esta cita de Bronowsy, "debemos darnos cuenta que la conectividad total de la naturaleza es algo que no puede ser imaginado en los sistemas axiomáticos”.

Esta cita me ayuda a definir dos ejes de lo que debe ser la educación. El primer eje es el mecánico. Pero como vemos, el eje mecánico no nos lleva más allá del pensamiento ni de la concepción que, en este ejemplo, tenían Newton y Leibniz. Así, para ir más allá del pensamiento y las concepciones de Newton y Leibniz se necesita algo más que el mecanicismo, se necesita de imaginación. Y la imaginación no es mecanicista.

El progreso científico, mediante la observación y la experimentación, como procesos de descubrimiento, ha sido organizado en programas estructurados de investigación, hay una mecánica en ello, y es a través de esos procesos mecanicistas que descubrimos las cosas.

Pero hay otro eje: crear. Crear desafía esa estructuración tradicional, no hay una mecánica para ello. Y dado que no hay un enfoque mecanicista para cultivar la ingeniosidad, que es la que nos hace crear, lo que nos queda es la opción de desarrollar esta capacidad en asociación con aquellos que puedan estimularla; es ahí donde entra el profesor.

En otras palabras, tenemos dos ejes intelectuales que debemos desarrollar. Un primer eje es el de la investigación y el descubrimiento, que requiere de un programa bien organizado: ir a las clases, leer los libros, ser enseńado a multiplicar, recibir los términos de una ecuación, poder despejarla, y todo esto bien estructurado. Es como en el caso de un pintor a quien le enseñan toda la mecánica de cómo se usan los pinceles, cómo se combinan los colores y demás técnicas apropiadas; pero entonces hay otra parte de la historia del pintor, en la que le dan un lienzo en blanco y el pintor tiene que pintar algo en ese lienzo, y pintar algo es crear un algo y eso no se enseña. La única forma de aprender es, por ejemplo, ser aprendiz en el laboratorio de Miguel Ángel, y es por esto que el profesor es sumamente importante; porque el profesor no es solamente una persona que ayuda al estudiante en el proceso mecanicista, sino que tiene también que estimular esa otra dimensión, trabajar en ese otro eje, el de la ingeniosidad y la creación, que es el eje del que quiero hablar. 
Aquí planteo una pregunta: ¡estamos haciendo todo lo que nosotros podemos como educadores? Y mi respuesta a esa pregunta es que no. Lo que nosotros hacemos muy bien es desarrollar disposición y capacidades intelectuales. Nosotros enseñamos, no a indagar de verdad; enseñamos el análisis, la reflexión, el pensamiento crítico, y todo esto corresponde al primero de los ejes que he identificado, que es el eje de conocer, el eje del saber. Para saber se va a la universidad, y cuando se sale de la universidad la persona sabe, algunas mucho, otras poco, pero al fin y al cabo se trata de saber, eso es lo que uno aprende leyendo libros, asistiendo a clases, cursando las "materias".

Para proseguir en esta discusión de cómo es que aprendemos, quiero auxiliarme de la gráfica que presento debajo. Partimos de un dominio, representado por el óvalo central en el gráfico, que se estudia a través de una o más "materias". Digamos, por ejemplo, mecánica clásica o mecánica cuántica, o evolución de las especies. Entonces tenemos un programa estructurado del dominio o de la "materia" que uno debe desarrollar. Empezamos por lo básico con nuestra indagación e investigación, y seguimos avanzando hasta que el trayecto se va haciendo más difícil y demandante, en esta trayectoria aparecen los "problemas", y cada vez que hay un problema, nos enseńan y aprendemos a navegar esos pasajes difíciles; hablamos aquí de "solución de problemas". Posteriormente llegamos a una frontera: regiones donde el conocimiento enfrenta sus límites, no existe todavía como tal (representada por un espacio en blanco al interior del óvalo, en la gráfica). Cualquier avance que hagamos aquí es merecedor de un ¡Eureka! Pues implicará que descubrimos algo. Es decir, llenamos ese hueco que existe en el conocimiento del dominio.

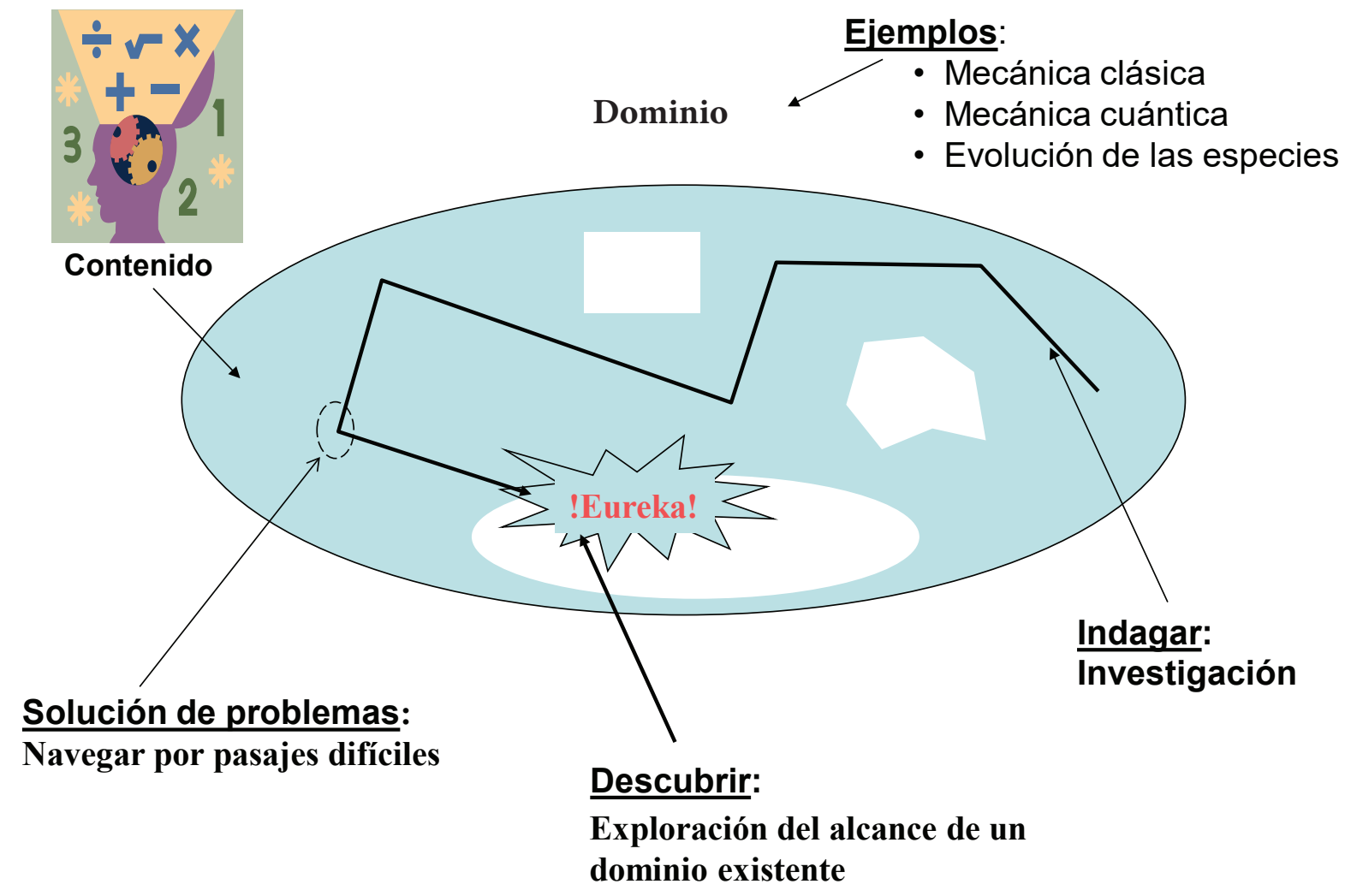


Hasta aquí nos mantenemos enfocados en contenidos, contenidos que enseñamos a los estudiantes, al igual que les enseñamos las trayectorias por esos dominios, y nosotros, los profesores, como investigadores, nos encontramos con esas regiones que están vacías, entonces descubrimos cómo "llenar" ese vacío. Ejemplos que a menudo ofrezco son los "vacíos" en la mecánica cuántica, en la mecánica clásica o en la evolución de las especies, dominios en los que hay "huecos" en el conocimiento, que se llenan a través de la investigación. Así, en uno de esos ejemplos, cuando se investiga al interior del dominio delimitado por las leyes de la mecánica de Newton, descubrimos que el movimiento real del planeta Urano se desviaba considerablemente de la órbita predicha por la teoría de la gravedad de Newton, y se conjeturó podía deberse a las perturbaciones ejercidas por otro planeta desconocido más lejano, llegando así al descubrimiento de Neptuno (mismo patrón que llevaría posteriormente al descubrimiento de Plutón).

Pero la pregunta es ¿y quién desarrolla un nuevo dominio? En ese sentido, otro ejemplo que siempre ofrezco a los estudiantes es que por más que se estudiaba cómo hacer un bombillo nuevo, distinto, la persona que estaba estudiando cómo hacer un nuevo bombillo nunca hubiera podido inventar una lámpara fluorescente, porque si trabajaba con ese norte, su tarea sería la de descubrir cómo se hacía un mejor filamento del bombillo, cómo contar con un mejor alambre, con más resistencia, pero nunca se plantearía lo que sería una lámpara fluorescente, sin filamento, y eso es ya un nuevo dominio.

Entonces crear, es la apertura e introducción de un nuevo dominio. Como intento ilustrar en el gráfico que presento debajo, implica una ruptura, salir del dominio (o los dominios) existentes y empezar uno nuevo. Es un acto de ingeniosidad y eso no existe tradicionalmente en el currículo. En el currículo, lo que le ofrecemos al estudiante es indagación y análisis, y reflexión y pensamiento crítico. Pero hay que ir más lejos y es por eso es que el profesor es sumamente importante.

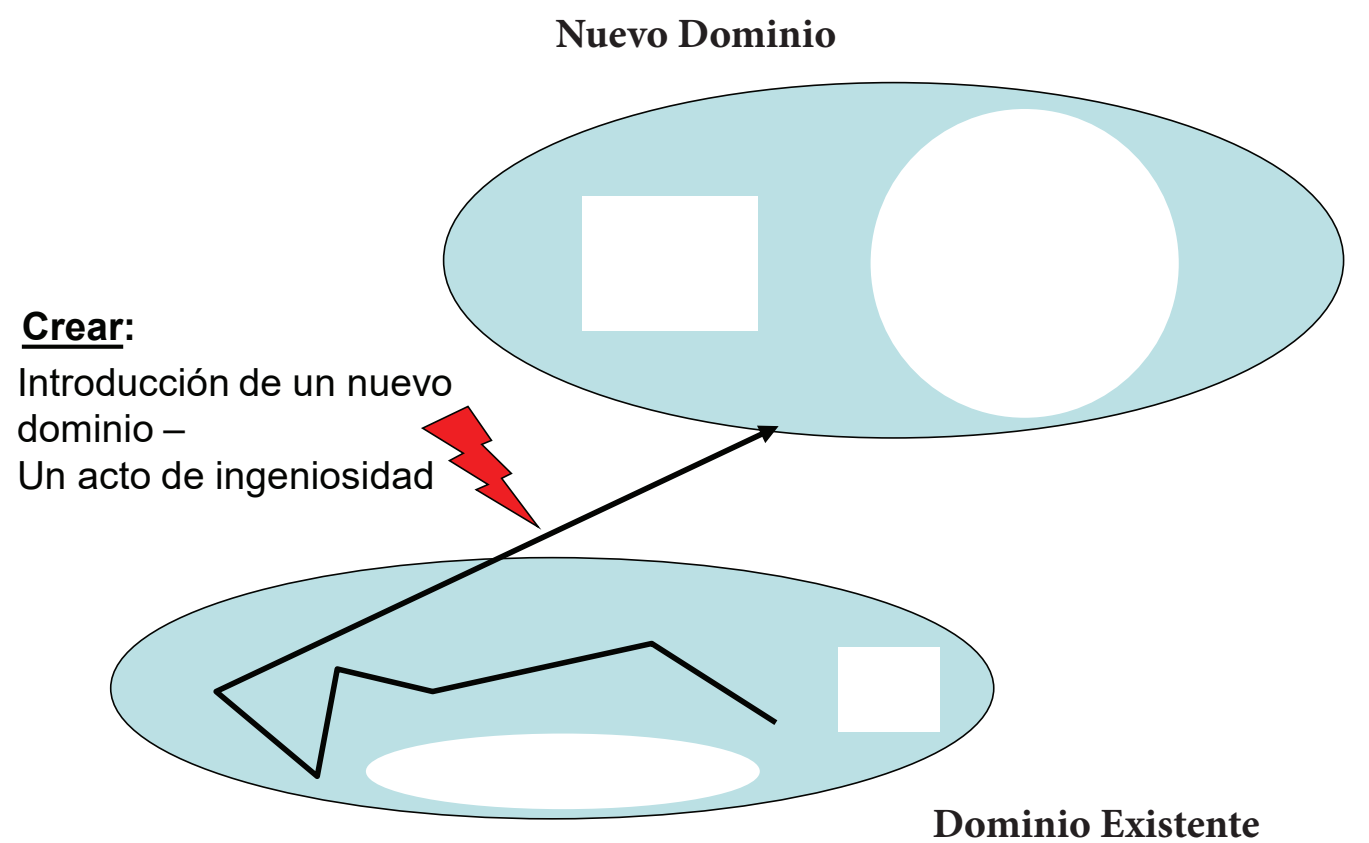


Volviendo entonces a la pregunta que hice primero, ¿cuál es el propósito de la educación?, y a la respuesta tradicional de que su propósito es desarrollar el intelecto, cultivar la mente, he planteado que eso quiere decir dos cosas: por una parte, desarrollar la disposición y las capacidades intelectuales para indagar y analizar críticamente, pero también para crear. Y esto último no se aprende en los currículos que tenemos, no tenemos como aprenderlo de libros, sino que se aprende más bien por medio de la exposición a los valores de los profesores, los valores del entorno, el entorno institucional de la academia, es decir la comunidad académica.

Mi observación es que el paradigma vigente de la educación favorece el desarrollo de una disposición a indagar y a analizar críticamente la creación. En otras palabras, la creación de Dios, o la de otra persona, pero está en déficit respecto de desarrollar la disposición a efectivamente crear por sí mismo. Aquí es oportuno hacer la siguiente cita de Emerson quien nos dijo que: "No les enseńamos a aspirar a todo lo que pueden ser. No les damos un entrenamiento como si creyéramos en su naturaleza noble", la naturaleza noble del estudiante, por supuesto.

Entonces, como hemos visto, la educación tiene en sus propósitos dos ejes y en estos se puede ir de cero a lo máximo; todo el mundo no tiene que ser como Newton o cualquier otro genio como él. Alguien puede crear sin que su creación sea una de orden deslumbrante, pero si es creación, en la magnitud que lo sea, es un acto que es completamente diferente al saber. Yo les digo a los estudiantes, en mis clases, que en este eje ellos y yo somos completamente iguales, porque por más que yo pueda saber, un acto fuera de ese eje es un acto independiente de lo que yo sé, y en esa forma yo estimulo a los estudiantes para que sepan que en este eje de la creatividad y la creación necesitan más que solamente saber y que, por más que yo sepa, ellos y yo somos completamente iguales.

Mi argumento es que toda persona ha sido dotada de ingeniosidad y de la capacidad de crear; ciertamente, algunas personas son más ingeniosas que otras, todo el mundo tiene esa capacidad. Entonces el profesor, con este concepto, tiene que hacerse como el conductor de una comunidad académica que es más amplia en sus intereses, que solamente lo que está pasando en una clase circunscrita a una "materia". Y tiene que proceder a desarrollar las conexiones entre las disciplinas.

Una de las peores cosas que encuentro en la educación es que las materias están completamente fragmentadas y compartamentalizadas. El que está enseñando literatura no tiene idea de lo que se está enseñando en matemáticas; el que está enseñando matemáticas no tiene idea de lo que se está enseñando en historia, y así las materias se enseñan como si cada una tuviera un contenido completamente aislado de todo lo demás. Los estudiantes de física o de ingeniería, por ejemplo, preguntan por qué deben tomar cursos de historia, para qué deben tomar cursos de literatura. Para un estudiante de tercer año en esos campos tener que cursar historia es como si se le estuviera enviando a Afganistán. A esos estudiantes les digo que si quieren ser un robot, entonces lo único que tienen que aprender es lo estrictamente correspondiente a su área técnica, pero que un robot lo puedo yo comprar ahora mismo en cualquier parte del mundo bien barato. Hoy en día puedo ir a la India o a China y "adquirir" un ingeniero por casi nada. Pero si quiero a alguien ingenioso, creativo, no es tan fácil conseguirlo. Y para desarrollar el ingenio y la capacidad de crear se necesita de la historia, de la literatura y de otras "materias" que nos ayuden a desarrollar la capacidad de crear. Entonces, con ese marco de referencia, podemos desarrollar las conexiones al interior y entre las disciplinas, de manera que los estudiantes aprendan a ver la educación como un proceso completo, coherente e interrelacionado; el marco de referencia para explorar al interior y a través de los temas. Porque para mí uno de los ingredientes fundamentales para crear es la multi-dimensionalidad del conocimiento.

\section{Hacia un currículo funcional}

Aquí entra el segundo ámbito que quiero examinar: ¿qué, en verdad, es un currículo? ¿Cómo proveemos los medios para el desarrollo intelectual que abarque los dos ejes de los que hemos hablado?

Si estamos de acuerdo en que el propósito de la educación comprende los dos ejes que hemos identificado, entonces el papel de esta concepción es el de 
guiar el desarrollo de los currículos y de los cursos y oportunidades de aprendizaje y desarrollo que se consignen en los mismos. Partiendo de esto, ahora podemos plantearnos cuáles serán los cursos o "materias" a incluir. Pero también hay que pensar en otras actividades y oportunidades diferentes en las que los estudiantes deben participar en la universidad. Unas actividades serán en cursos, otras, desarrolladas en los cursos también, pero no sentados en un aula, sino expuestos a situaciones de mayor interacción con los profesores. Podemos organizar y debemos organizar actividades especiales, como proyectos interdisciplinarios. Hay muchas cosas que debemos empezar a pensar con esta nueva perspectiva, con este nuevo concepto, de que tenemos dos ejes que desarrollar.

Un fallo en el diseño de un currículo funcional, que sea verdaderamente funcional, es que por tradición se brinda más atención a los contenidos y a los cursos que debe incluir y no a qué es lo que estamos desarrollando en el estudiante. Por supuesto que hay que incluir los cursos y sus contenidos, pues los estudiantes tienen por delante mucho por aprender. Pero quiero explicarme respecto de mi insistencia en los propósitos de la educación. Quiero ilustrar mis preocupaciones con un ejemplo. Cuando era decano de artes y ciencias había una profesora de literatura a quien le encantaba que los estudiantes en su curso leyeran seis, ocho y hasta diez libros; al saber esto yo reaccionaba diciéndole: "Profesora, ¿para qué quiere usted que esos muchachos lean diez libros?; mire, enséńeles a leer un solo libro con tanto interés, con tanto gusto, que por el resto de su vida no solamente leerán ese, sino que leerán cientos de libros". Lo que estaba en mi preocupación era preguntarme más por el propósito que por los contenidos. Me preguntaba: ¿tú sabes lo que es tener que leer seis, ocho o diez libros en un solo curso?, ¿para qué?, ¿solamente para que manejen más información y contenidos? El riesgo es que el estudiante salga de ese curso diciendo: nunca en mi vida quiero yo saber de literatura, y esa no es la idea.

Debo insistir en la cuestión de proveer los medios para el desarrollo intelectual. Y lo voy a hacer en el contexto de la tecnología, porque para mí la educación en la tecnología es la que podría ser más completa en ambos ejes principales de la educación. Mantengo un debate con mis colegas en lo que se llama Artes Liberales, porque ellos, en las Artes Liberales, siempre han dicho que son los que más hacen, los que más ayudan a desarrollar la mente. $Y$ yo les digo que si bien hubo un tiempo cuando las Artes Liberales eran la forma más propicia y completa de cómo desarrollar la mente y el intelecto, ahora lo es la tecnología. Entiendo que la tecnología moderna es la que ofrece una mejor plataforma para la educación en la creación, como ningún otro ámbito, como ninguna otra "materia".

¿Qué es la tecnología moderna? La tecnología moderna ahora tiene cuatro dimensiones. Usualmente uno piensa en tecnología como artefactos, eso es lo que usted piensa cuando dice tecnología y piensa en su celular, su carro, su televisión, es decir, en gadgets, instrumentos, dispositivos, artefactos. Esa es una dimensión de la tecnología. Pero la tecnología moderna es mucho más que eso. Porque la tecnología también re-define lo que es ser humano. Ese planteamiento no es mío, viene de pensadores como Heidegger y Habermas, quienes pensaron y escribieron sus reflexiones sobre esa nueva dimensión de la tecnología. Y es sobre la base de esas reflexiones que entro en debate con mis colegas de las Artes Liberales, a quienes les digo: "señores es tiempo que ustedes repiensen los griegos, ya estamos en un nuevo milenio, ya estamos en el Siglo xxi y debe haber uno de ustedes, filósofo o pensador, que empiece a repensar los griegos, porque hemos evolucionado ya por medio de la tecnología en un ser humano que es muy diferente al ser humano de hace mil años”.

Permítanme aquí referirme a otras dos dimensiones de la tecnología, recordarles de ellas. Primero, la tecnología moderna no es igual a la ciencia. En ciencia nosotros exploramos lo que Dios creó, el universo; estudiamos el universo y queremos tratar de entender lo que es. Pero les quiero recordar que nosotros estamos bien lejos de verdad de poder ver lo que Dios creó. Lo que nosotros tenemos son teorías y marcos de referencia o plataformas conceptuales; hemos creado plataformas conceptuales para ayudarnos a entenderlo. Pero esas plataformas no son de verdad lo que Dios creó, son plataformas que nosotros nos hemos inventado. Y aquí tenemos otra dimensión 
de la tecnología. Las plataformas conceptuales nos ayudan muchísimo en tanto y cuanto hemos podido hacer televisores, carros, aviones, cirugías, etc. Pero eso es distinto de saber qué fue lo que Dios hizo en el universo, y esas cosas que nosotros hacemos no son lo que Dios hizo.

Lo que nosotros hemos creado, basándonos en nuestras teorías, marcos de referencia y plataformas conceptuales, constituye un mundo propio, por sí mismo. Déjenme tomar la teoría cuántica como ejemplo. La teoría cuántica nos ofrece una visión del universo, para muchos bastante extraña, pero la teoría cuántica tiene también muchas dimensiones que tal vez no tienen nada que ver con lo que Dios hizo. Y ahora podemos investigar sobre la base de esa plataforma conceptual que nosotros hemos creado; es decir, podemos hacer ahora complementos a la naturaleza para explorar el alcance de los marcos de referencia, de las plataformas conceptuales que nosotros hemos inventado. A eso le llamamos ahora tecno-ciencia, una tercera dimensión de la tecnología. Y como parte de la tecno-ciencia, podemos, por ejemplo, hacer cosas de las dimensiones de un átomo, y en esas cosas de dimensiones de un átomo, suceden otras cosas. En otras palabras, podemos ahora hacer tecnología donde suceden cosas que nosotros podemos usar para explorar el grado de alcance de las teorías que nosotros nos hemos inventado. Porque esas teorías tienen alcances que no necesariamente se relacionan a lo que de verdad pasa en la naturaleza, y esas nuevas creaciones solamente existen ahora en la historia del mundo, del universo.

Otra dimensión de la tecnología, la última de las cuatro de las que quiero hablar, pero que para mí es la más importante, consiste en qué podemos imaginar más allá de las fronteras de lo que pudo ser imaginado; podemos suponer que hay un corpus de todo lo que nosotros sabemos, pero siempre hay una persona que puede imaginar más allá de eso. ¿Y quién es esa persona? Se dirá que esto es competencia de genios, pero en esto es mejor pensar en un continuo. Y en ese continuo nadie sabe quién puede llegar a imaginar cualquier cosa si se le estimula el ingenio, a la creación. De cualquier persona estimulada puede resultar el imaginar algo más allá de la frontera de todo lo que nosotros nos hemos imaginado. Esto no es ya cuestión de que una persona sea un genio, no; se trata de un continuo de posibilidades y cualquiera puede ser estimulado y desarrollado para que pueda llegar a ser imaginativa, creadora, ingeniosa, en un grado u otro.

Es por esas cuatro dimensiones de la tecnología moderna que la educación en un instituto de tecnología se hace más exigente que en cualquier otro ámbito de formación en el mundo académico y que puede convertirse en una excelente plataforma para el desarrollo de los dos ejes de los que he hablado: el de análisis y el de la creación.

$\mathrm{Al}$ respecto, una de las primeras cosas que debemos hacer como profesores es evitar los estereotipos. Nosotros, antes siempre decíamos que los científicos descubren, que los artistas crean y los ingenieros resuelven problemas. Pero si asumimos la perspectiva que proponemos, la creación no es patrimonio particular de ningún grupo, sino una potencialidad y una capacidad a desarrollar en todos ellos. Decimos que los ingenieros solucionan problemas, pero entonces la pregunta que yo hago es: ¿y los creadores que hacen? Entonces lo que debemos hacer es olvidarnos de todos esos estereotipos y concebir un currículo en el que trabajemos los dos ejes de propósitos educativos con todo el mundo.

\section{Rol del profesor en el proceso educativo}

Para terminar esta plática quiero referirme al tercero de los ámbitos que mencioné al principio. ¿Cuál es el rol del profesor en el proceso educativo? Para mí el profesor es el componente singular más crítico para la implementación de cualquier modelo educativo. Desde cuando era decano vengo diciendo que nadie es más importante en la educación que el profesor, ni presidentes, ni expertos o ninguna otra persona. El profesor en interacción con el estudiante es la educación. Y si el profesor no tiene la disposición a conducir y fomentar y propiciar el logro de los propósitos de la educación, en los dos ejes que hemos identificado, por más bonitas que sean las aulas y por más recursos de los que se disponga, por más cursos y clases y más libros y contenidos, no obtendremos los resultados que buscamos. Si el entorno, el ambiente, 
la comunidad intelectual no promueven y tienen ciertos valores que puedan propiciar el desarrollo en los dos ejes fundamentales, para mí, la educación estará en falta. Este hecho es particularmente subrayado en esta concepción de la que les estoy hablando, con sus dos ejes, puesto que el desarrollo de la capacidad de inventar requiere mucho más que las normas y formas tradicionales de la educación. El profesor debe ahora convertirse, digamos, en alguien como Miguel Ángel, no solamente en lo técnico, sino en la forma de ser.

Lo más importante es que la experiencia de educación sea multidisciplinaria e interdisciplinaria. Que todos, quien enseña matemáticas como quien enseña ingeniería mecánica, quien enseña ingeniería civil y quien enseña historia, contribuyan a hacer ver las disciplinas como parte de una totalidad. ¿Y cómo hacemos eso? Una estrategia es la de enseñanza en equipo o team-teaching. Puedo ofrecer un ejemplo en términos de una experiencia en uno de mis cursos. Imparto un curso de primer año de física y la razón que me motiva a conducir ese curso es porque me ofrece la oportunidad de introducir a los estudiantes a una forma de comportarse y pensar que implica más que simplemente sentarse en una silla a oír a un profesor hablar y disertar. Lo que hago en este curso es invitar a colegas profesores en historia y en literatura a que participen conmigo en sesiones de esta clase, y mientras estoy tratando el tema de la electricidad y el magnetismo, por ejemplo, pido a mis colegas que me ayuden a explicar lo que era el mundo cuando vivía Maxwell, quien formuló las ecuaciones que describen el electromagnetismo. Y así, también como ejemplo, cuando estudiamos las contribuciones de Leibniz a la física, tomando en cuenta que Leibniz era un filósofo, invito a un profesor de filosofía al curso y le pido que nos cuente de Leibniz como filósofo y nos ayude a ver cómo su pensamiento filosófico conectó con sus aportes a la física. Pero también invito a un profesor de historia para que nos hable del mundo que le tocó vivir a Leibniz y cómo era la vida de este; la expectativa con todo esto es que los estudiantes no vean a Leibniz como un genio lejano, sino como una persona que, al igual que los estudiantes, enfrentaba los asuntos de la vida cotidiana; que también compren- dan cómo era el mundo alrededor de Leibniz, qué estaba pasando y cómo todo eso tuvo que ver con sus aportes.

Con los ejemplos anteriores he querido ilustrar cómo intentar que nuestra aproximación a los temas abordados sea una experiencia educacional multi-disciplinaria e inter-disciplinaria y cómo la enseñanza en equipo o team-teaching puede ayudarnos a ello.

Al iniciar mi plática dije que brindaría algunos ejemplos. Acabo de hablarles acerca de uno, pero ahora quiero referirme a una experiencia que tenemos aquí en New York University: el Fórum. Todos los estudiantes de primer año tienen que participar en el Fórum. Reunimos en el Fórum aproximadamente 500 estudiantes. El Fórum es un curso, pero no es un curso tradicional. A este curso invitamos personas de todas las áreas, campos y disciplinas: políticos, pintores, gente de negocios, inventores, ingenieros, técnicos... Estos invitados vienen cada quince días, es decir, una semana sí, otra no, y participan en encuentros con los estudiantes del Fórum en un auditorio. Cada invitado tiene la misión de compartir con los estudiantes presentándose, explicando quien es, qué estudió, en qué se interesa, contando que es lo que está haciendo.

La semana siguiente, a la visita de cada invitado, es una semana de debate. Dividimos a los estudiantes en grupos de 20 y cada uno es dirigido por un profesor. Y cada grupo debe discutir sobre lo que ha hecho y hace el conferencista invitado la semana previa. La discusión gira acerca de la persona, su trayectoria, lo que hizo y está haciendo, sus testimonios, sus ideas y planteamientos. Como ven, la idea con el Fórum es que los estudiantes se vean a sí mismos respecto de las personas invitadas, viendo que en el mundo real esas personas hacen cosas que van más allá de lo que aprendieron en sus estudios, en sus clases, en un curso, y que en la vida se necesita más que solamente análisis.

Otra experiencia muy importante a las que nos hemos abocado en New York University es una un tanto más problemática y consiste en re-pensar los requerimientos de graduación. Nosotros, al asumir las ideas que les he presentado en esta plática, de que debemos desarrollar el intelecto en dos ejes, el de la 
investigación, la indagación y el análisis, y el del ingenio y la creación, nos planteamos ser consecuentes con esa concepción de los dos ejes; entonces, para saber si ambos se han desarrollado en los estudiantes debemos requerir algo más que solamente cuáles y cuántos cursos fueron aprobados y qué calificación se obtuvo en esos cursos. Eso es lo tradicional. Pero ahora nosotros tenemos un nuevo requerimiento; este nuevo requerimiento consiste en que cada estudiante, al finalizar los cuatro años de universidad, al concluir su grado, debe presentar al departamento al que pertenece un portafolio de las cosas que el estudiante ha originado durante esos cuatro años. De esta manera tenemos un grupo de actividades paralelas a los cursos y estas actividades brindan oportunidades para que los estudiantes puedan desarrollar esas otras realizaciones que deben presentar a sus departamentos durante los cuatro años de sus estudios. Por ejemplo, ahora mismo tengo dos estudiantes de primer ańo que trabajan conmigo durante el verano. Estos estudiantes pasan el verano investigando, como lo hacen otros estudiantes; cada profesor trabaja con uno o dos estudiantes, yo tengo dos. En mi caso acostumbro a debatir con ellos dos o tres veces a la semana sobre sus trabajos de investigación y así, al fin del verano, ellos tienen que mostrar lo que han logrado. De esa manera lo van acumulando cada año para, al cabo de los cuatro años, tener un portafolio como si fueran artistas, y presentarlo al departamento.

Hay otro asunto muy importante para ayudar a los estudiantes en su desarrollo en este nuevo eje educativo y es el de la reacción y manejo del fracaso. Todo el mundo le tiene miedo a fracasar, porque si usted fracasa teme que todo el mundo se ría. Pero hay que saber vivir teniendo el fracaso como una posibilidad y sabiendo reaccionar ante el mismo. Siempre les cuento a mis estudiantes sobre un artículo de opinión que escribí en el New York Times y les digo que cuando yo publiqué ese artículo, me escribieron miles de cartas y que, de todas esas cartas, usualmente noventa y nueve reaccionaban en contra de lo que yo decía y solo una estaba de acuerdo conmigo; entonces un amigo me dijo: "Mira Erich, toda esa gente que está en contra de lo que opinaste te hace un honor, porque por alguna razón te escribieron, si no les hubiera importado nunca te hubieran escrito". Con esto lo que quiero ilustrar es... ¿por qué le debo tener miedo a fracasar? ¿Qué hay de malo en fracasar? Y por eso le digo a mis estudiantes: Mira si tú nunca fracasas, tú nunca vas a saber qué es lo que es eso, pero probablemente tampoco sabrás lo que es el éxito. Lo que hay que entender es que el fracaso no es absoluto ni definitivo, para lo cual debemos saber cómo reaccionar con los estudiantes cuando enfrentan un fracaso y facilitar que lo encaren y sepan reaccionar ante él y así se logra que frente al mismo se recuperen y sigan hacia adelante. Por eso todo este asunto del manejo del fracaso es otro de los ámbitos en los que es importante ayudar al estudiante y eso no se puede hacer simplemente en una sesión de clase.

Antes de terminar quiero ofrecer otros ejemplos de las cosas que podemos y debemos hacer. Sobre un primer ejemplo ya les hablé al contarles cómo son presentadas las asignaciones de clase enseñando en conjunto con profesores de otras ramas.

Otro ejemplo que puedo ofrecer es lo que hago con en mi clase de física para estudiantes de primer y segundo ańo, especialmente con los estudiantes de ingeniería. Lo que ellos quieren es que le presente las ecuaciones para saber qué es lo que tienen que hacer para pasar el examen y avanzar y terminar sus programas y obtener sus diplomas que les permitan irse a hacer máquinas y otras cosas. Entonces me molesto y les respondo en estos términos: "Mire, seńor, a un tipo como usted yo lo voy a adquirir en la India por diez mil dólares, bien barato, o en cualquier otro lugar donde produzcan muchos ingenieros". Y es que yo no le pongo mucha atención a escribir fórmulas en una pizarra, y a muchos estudiantes no les gusta eso, porque en lugar de insistir en las fórmulas los invito a pensar y muchos están circunscritos a una cuestión mecánica: ¿qué es lo que estará en el examen?, ¿qué es lo que necesito para aprobar este curso? Entonces es importante que cambiemos cómo presentamos las asignaciones, de modo que propiciemos el pensamiento creativo. Y por eso, en mis clases, paso mucho tiempo hablando de las circunstancias que llevaron a una persona a crear lo que estamos estudiando. Lo hago con dos propósitos, primero, desmitificar a las personas que han hecho todo eso, para que el estudiante 
no las conciba como personas extrańas, de otro planeta; en segundo lugar, porque tal vez todo lo que estamos estudiando ahora es, a fin de cuentas, erróneo, en el sentido de que están trabajando bien para nosotros por el momento, pero... ¿quién puede decir si esa es la forma en que vamos a concebirlo y a hacerlo dentro de cien años? Mi papel es hacer sentir a los estudiantes que ellos pueden cuestionar todo lo que estamos aprendiendo, y estimularlos a participar en actos de invención, con grados crecientes de originalidad e independencia

Otro ejemplo lo constituyen los estudios de casos. En cada curso que imparto, para cada tema que estudiamos, tomamos siempre ejemplos o casos que podemos usar para que entonces los estudiantes se den cuenta de cómo lo que estamos estudiando se ha desarrollado desde el principio hasta llegar a la situación presente y cómo está afectando ahora mismo a la humanidad. En otras palabras, referir los materiales de la clase a su contexto más amplio. Así, empleamos los estudios de casos que sean de interés para el estudiante, para permitirles la experiencia del acto de creación y considerar su impacto sobre la sociedad y el intelecto. Empleándolos, nos asistimos para ayudar a los estudiantes a enfocarse en la evolución, los procesos y productos de las invenciones, para proveerles con oportunidades para apreciar y desarrollar por ellos mismos la capacidad de crear y transformar la vida. Ellos nos permiten subrayar la manera como los humanos pueden iniciar el cambio, sea este para bien o para mal, y examinar las consecuencias de lo que hacemos. También para enfatizar la importancia de investigar los desarrollos alcanzados por la gente a través del espacio, el tiempo y las culturas. Empleándolos nos proponemos ir más allá del acto de creación mismo, guiando a los estudiantes a examinar la experiencia y a reflexionar sobre el proceso creativo.

Mi último ejemplo tiene que ver con la asignación del proyecto personal. Cada estudiante completa un proyecto personal, un trabajo en un área de su interés, que sea un intento de "mejoramiento" en el 'estado del arte', en el que demuestre ingeniosidad. Cada proyecto debe reflejar un entendimiento personal del tópico. Se espera de los estudiantes que elijan su proyecto, que puede tomar muchas formas, desplieguen el proceso hasta su término en "colaboración" con el profesor y que, al final, el mismo sea presentado a "pares".

Permítanme decirles que entiendo que esto no lo hemos hecho muy bien; la forma como hacemos aquí el proyecto personal no me gusta y eso lo estuvimos debatiendo la semana pasada. Aquí todos los estudiantes del cuarto ańo tienen que hacer un proyecto, pero solamente se hace en ese año. Y la razón por la que no me gusta es que debemos tener un proyecto que empiece desde el primer año y el estudiante cada año le sume un poco al proyecto, y tal vez, entonces, a lo largo del tiempo, el proyecto tome nuevas dimensiones, pero lo importante es que sea un proyecto que sirva como un vertebrador por los cuatro ańos y que le dé estructura a esa noción y a esa actividad de crear.

De todas maneras, las asignaciones de proyectos son muy interesantes. Tengo unos estudiantes de primer año que inventaron una pelota de tenis para jugar de noche, ahora ellos se han vuelto locos con esto porque una persona quiere usar su invento para un negocio. Tengo otros estudiantes con los que empecé en su primer año con el desarrollo de un rayo láser en frecuencias de Terahertz, una región del espacio electromagnético que no hemos explorado suficientemente y en la que no tenemos, ahora mismo, fuentes aprovechables de energía. Otros estudiantes están trabajando en cuestiones de espacio-tiempo en las mecánicas clásica y cuántica. Tengo también un grupo de estudiantes empezando a trabajar en la estabilidad del átomo. Y así, entonces, hay muchos proyectos que le damos a los estudiantes para que ellos empiecen a desarrollar.

Esos son algunos de los ejemplos del tipo de cosas que podemos hacer para empezar a explorar esa dimensión del desarrollo intelectual que es más que analítico, cultiva la capacidad de crear y espero me hayan ayudado a hacerme entender. 